\title{
Le jeu de la création : Le mangeur (2005) de Ying Chen
}

Nicole DUNHAM, State University of New York (SUNY) at Buffalo, Etats-Unis

Dans l'article « À mi-chemin entre deux mondes : Parcours féminins chez Ying Chen », Gabrielle Parker écrit : «De fait, aucun des romans [de Ying Chen] paru à ce jour n'a une fin : il s'agit d'épisodes qui peuvent se lire indépendamment sans doute, mais sont aussi 'à suivre' : le dénouement est encore à venir » (78). Le mangeur est le cinquième roman dans cette suite d'épisodes. Dès la première phrase du roman, l'idée de la fin se présente comme hantise. Pourtant, cette même phrase contient la remise en question de la possibilité d'une fin définitive : «Jusqu'à la fin, celle de mon père et la mienne [...] avant ce singulier après-midi où tout [...] s'était défait, et par conséquent était entré en un ordre nouveau [..], mon père et moi nous avions mené une vie à peu près normale » (7). La fin n’est pas une fin définitive, donc, mais la transition d'un ordre à un autre.

L'action du Mangeur se déroule dans deux espaces-temps, deux ordres, différents : celui où la narratrice habite chez son père artiste et celui où celle-ci est l'épouse d'un archéologue désigné par la lettre A. Comme les autres romans de Ying Chen mettant en scène cette narratricespectre, il s'agit d'un récit raconté à la première personne, avec des chapitres alternant entre les événements dans l'espace-temps du père et celui de A. Dans l'espace-temps du père, la fille et le père souffrent d'une étrange maladie liée certainement au fait que l'arrière-grand-mère de la narratrice avait eu une relation conjugale avec un poisson. Cette maladie provoque un appétit inapaisable chez le père, qui finit par avaler sa fille unique l'après-midi où elle attendait un ami pour aller au cinéma. Pourtant, la narratrice n'en meurt pas, et se retrouve dans le train où elle rencontre A., qu'elle épousera par la suite. Un jour, le couple marié va au vernissage d'un artiste disparu dans des circonstances mystérieuses, donnant une plus grande valeur à ses œuvres. Pendant ce vernissage, la narratrice se rend compte que ce peintre avait été son père dans une vie passée. Elle décide de se rendre à l'ancienne maison du peintre pour mieux comprendre les évènements de cet après-midi au cours duquel elle aurait dû mourir. Inquiété, A. vient chercher celle-ci à la maison du peintre et la ramène à la maison, en présentant ses excuses à l'inspecteur venu faire une enquête sur les circonstances de la disparition du peintre. 
Au cours du roman, une équivalence s'établit entre la fenêtre de la maison du père, l'appareil digestif de ce dernier, ses tableaux et l'espace-temps de A. Étudiés du point de vue des théories du jeu de James Hans et de Tilman Küchler, qui caractérisent le jeu comme une déconstruction structurante et un processus de perpétuelle remise en question, ces éléments deviennent des aires de jeu permettant la remise en question et le dépassement des concepts de l'intérieur et de l'extérieur (pour la fenêtre), du créateur et de son œuvre (pour les tableaux), du mangeur et de la proie (pour l'appareil digestif), et du passé, présent et avenir (pour la maison de A.). Chez Ying Chen, ces aires de jeu prennent la forme de tunnels. En nous servant du texte du même titre écrit par Ying Chen, nous avançons que cette notion du jeu s'applique également à l'ensemble de son œuvre.

Se situant entre la vie et la mort, le passé et le présent, l'humain et l'animal, la narratrice du Mangeur est un personnage spectral qui remet en question la pertinence de telles catégories. Cette absence de distinction, notamment entre le passé et le présent, est la source d'une grande tension entre la narratrice et A. qui, en tant qu'archéologue, veut absolument situer les événements selon un déroulement chronologique. Ses tentatives de se conformer aux règles de la logique mènent à un résultat de « désordre total » car elle essaie d'enfiler les épisodes sur « une ligne [qu'elle] espère chronologique mais qui ne peut pas l'être »(19). La chronologie est impossible chez la narratrice qui effectue sans cesse des va-et-vient entre le passé et le présent.

Rosalind Silvester démontre une remise en question systématique chez Ying Chen des catégories toutes faites. La narratrice de ses romans vit à la fois dans plusieurs espace-temps, lutte avec son double dans Querelle d'un squelette avec son double (2003), change d'espèce dans Espèces (2010) et se confond avec A. dans La rive est loin (2013). Les protagonistes de ses romans se situent «between the absolutes » et résistent le fixage de «their fluid identities with a more rigid view of boundaries between self and other » (Silvester 407). La narratrice ne parvient pas à localiser la frontière entre « les absolus » (la vie et la mort, le passé et le présent, l'humain et l'animal, le soi et l'autre) parce que son existence remet en question la distinction entre les deux.

Les définitions du jeu de Hans dans The Play of the World (1981) et de Küchler dans Postmodern Gaming : Heidegger, Duchamp, Derrida (1994) éclaircissent le sens de cette remise en question systématique puisque le jeu, selon ces théoriciens, est un processus de création basé 
sur un questionnement perpétuel. Küchler désigne le jeu comme « another name for the economy in which meaning is produced and from where it arises» (10). Pour Hans le jeu, «is a structuring activity, the activity out of which understanding comes. Play is at one and the same time the location where we question our structures of understanding and the location where we develop them »(x). Le jeu est une activité créatrice permettant la remise en question sans destruction et la création de nouvelles structures du savoir découlant d'anciens schémas et structures. En d'autres termes, la création est, selon Hans et Küchler, nécessairement lié à la fois au processus de la recréation, selon lequel le nouveau et refait à partir d'anciennes structures, et à la récréation dans la mesure où c'est un processus ludique.

Bien que Ying Chen n'évoque pas la création littéraire comme jeu, la figure du tunnel fonctionne à la manière du jeu défini par Hans et Küchler. Elle se sert de la figure du tunnel dans son essai intitulé «Le tunnel », composé en juin 2005, un an avant la publication du Mangeur. Ce texte apparaît dans un recueil de textes rédigés lors des ateliers de la littérature bipolaire, ALIBI, portant le titre: Alibi 2 : dialogues littéraires franco-chinois ${ }^{1}$. Le titre de Ying Chen détourne celui du recueil qui suggère conversation, échange, passerelle, pont entre cultures et entre traditions littéraires. Le pont implique la présence d'un obstacle. L'idée donc d'un dialogue littéraire franco-chinois présuppose un obstacle entre les deux éléments divisés par le trait d'union, créant une cavité entre les deux.

La construction d'un tunnel, d'un trou, rappelle le processus à la fois créateur et destructeur du jeu. Il relie deux éléments non pas par l'enjambement d'un obstacle, mais en creusant un trou permettant un passage souterrain. Pour construire un tunnel, il faut défaire la matière dans laquelle on creuse qui est à la fois la base de cette nouvelle structure. Par exemple, un tunnel qui relie deux villes séparées par une chaîne de montagnes dépend de la montagne pour sa structure. La montagne n'est plus un obstacle une fois le tunnel creusé puisqu'elle devient le support de cette nouvelle construction sans laquelle le lien entre les deux villes serait impossible.

Chez Ying Chen, cette aire de jeu qu'est le tunnel est le lieu privilégié de la création artistique. Le tunnel dont elle parle est ce qui fait le lien entre le monde extérieur et l'espace de l'écriture, décrit comme «pièce grise où [elle entend] le monde sans le voir » ressemblant « à un tunnel bien relié à l'extérieur et pourtant à l'écart » (118-19). L'écrivain creuse un trou et ouvre une brèche entre l'extérieur (les bruits du monde) et l'intérieur (la «pièce grise »). Au lieu de se 
servir d'un pont pour réconcilier l'extérieur et l'intérieur, ou dans le cas des dialogues littéraires franco-chinois, le monde francophone et la Chine, l'écrivain du tunnel crée un nouvel espace qui permet de rapprocher les deux éléments au lieu de les faire tout simplement dialoguer par le biais d'un trait d'union.

Ying Chen décrit le tunnel comme un espace à la fois relié à et à l'écart de l'extérieur. Cet espace ressemble à l'espace-temps de A. dans Le mangeur, où se juxtaposent les vies passées et futures de la narratrice. L'espace-temps de A., qui, dans d'autres épisodes, tels Immobile (1998) et Le champ dans la mer (2002), est évoqué sous des traits plus sinistres, devient dans Le mangeur un lieu de refuge, de paix et de recul parce que situé entre les vies passées et futures de la narratrice. La fenêtre, le ventre et le tableau sont tous les trois reliés à la maison de A., de même que le train par lequel la narratrice est arrivée : «Lorsque j’ai rencontré A. dans un train, il n'y a pas longtemps je crois, et l'ai suivi dans cette ville, j'ai sauté dans l'éternité. [...] En me hasardant sur les chemins les plus ténébreux [...] je porte en moi un temple qui est la maison de A. » (33-34). L'espace-temps de A. est un point fixe où se forme la juxtaposition avec les autres espaces-temps, ce qui lui permet de prendre du recul par rapport à ses vies passées et futures, ce qui lui permet de vivre dans une paix relative, sachant que ses excursions dans les vies passées ne seront pas permanentes : «[...] j'ai sauté dans l'éternité. Cela me convient, l'éternité. Cette fois, je ne mourrai plus » dit-elle (33). Malgré les tensions ressenties avec A. la narratrice se sent à l'aise dans la maison de $\mathrm{A}$. et l'espace-temps associé à cette dernière.

Une des images centrales du Mangeur, annoncée dès le titre, est l'appareil digestif du père, qui est un tunnel rapprochant le mangeur et sa proie, la narratrice. ${ }^{2}$ La maladie du père se manifeste sous forme d'un appétit insatiable. L'appareil digestif est un tunnel à travers le corps qui relie l'extérieur et l'intérieur du corps. Dans ce lieu de transformation, les aliments sont détruits par le processus de la digestion afin de créer de l'énergie, un processus de création par le biais de la déconstruction qui ressemble au jeu décrit par Hans et Küchler.

La figure du mangeur anthropophage apparaît les romans précédents de Ying Chen, notamment le premier roman mettant en scène une narratrice-spectre, L'ingratitude (1995), dans lequel la narratrice subit ses diverses appartenances comme des tentatives de dévoration de son identité, ce qui la mène finalement à une tentative de suicide. La narratrice de L'ingratitude dit, en parlant de sa mère : «J'avais parfois l'impression qu'elle avait envie de m'avaler vivante, de 
me reformer dans son corps et de me faire renaître avec une physionomie, une personnalité et une intelligence à son goût »(20). Elle essaie de trouver une issue dans sa relation amoureuse avec son fiancé Chun, mais ce dernier ressemble de plus en plus à sa mère, devenant un double de celle-ci, disant: «Je t'aime tant que j'ai envie de t'avaler tout rond » (110). Dans L'ingratitude, l'anthropophagie est évoquée au sens figuré pour exprimer une volonté de la part des «mangeurs » de façonner la narratrice selon leurs désirs au lieu de l'accepter telle qu'elle est.

La fonction de l'appareil digestif dans Le mangeur correspond à la définition du jeu que nous avons retenue, à savoir une activité à la fois destructrice et structurante, bien qu'il ne s'agisse plus de dévorer et de refaçonner l'identité de la narratrice selon une idée préconçue. Comme le jeu, ce processus sans fin, sans autre but que le jeu lui-même, la digestion est également un processus sans fin/faim. Le jeu n'est plus jeu s'il n'y a plus de remise en question des structures existantes et la digestion, source d'énergie nécessaire à la vie, ne peut s'arrêter que quand la vie n'existe plus.

Le dévorement n'aboutit pas à l'assimilation du corps de la fille dans celui du père mais dans une transposition dans le temps et dans l'espace. Au lieu de se retrouver dans le tunnel du ventre, elle change de tunnel en arrivant dans celui de l'espace-temps de A. Un saut dans l'espace et le temps qui n'obéit à aucune continuité logique ou chronologique car la narratrice se demande également comment il est possible de «sauter de la fenêtre de chez [s]on père et $[\mathrm{s}] \mathrm{e}$ faufiler dans le wagon occupé par A. » (44). Dans cet extrait, la narratrice dit qu'elle a sauté par la fenêtre, confondant fenêtre et ventre, deux lieux de passage et de transformation d'égale valeur dans l'univers du roman. La narratrice décrit cette juxtaposition comme une sorte de trou de ver : «Comme si cet après-midi était juxtaposé à l'autre, que ce temps dans son écoulement croisait un autre temps à cause de moi »(54). Son passage entre les lieux, les époques, les corps, les espèces (dans les autres « épisodes ») rendent toute chronologie, toute catégorisation, impossible.

La fenêtre occupe un rôle analogue à l'appareil digestif dans Le mangeur, reliant non pas mangeur et proie mais l'extérieur et l'intérieur de la maison. L'image de la fenêtre est également présente dans plusieurs romans de Ying Chen, notamment dans les romans suivant la publication du Mangeur comme Un enfant à ma porte (2008) où la narratrice installe une grille sur la fenêtre de la maison pour empêcher son fils adoptif de s'échapper et Espèces (2010) où la narratrice se 
transforme en chatte, la fenêtre devenant un lieu privilégié pour ses va-et-vient entre la maison et le monde extérieur, ainsi qu'un poste d'observation du voisinage.

Dans Le mangeur, la fenêtre relie la narratrice au monde extérieur : de la fenêtre, elle peut contempler le chemin menant à la ville et observer la patronne de l'épicerie. De manière inverse, l'ami de la narratrice arrive par ce chemin, et la patronne de l'épicerie voit ce qui se passe dans la cuisine de la maison du père. La fenêtre est également liée à la mort de la narratrice, car c'est « debout à la fenêtre [de la maison] » qu'elle comprend «pourquoi et comment ne pas vieillir », suite à la prédiction de son père qu'elle ne vieillira pas (10). La posture de la narratrice dans ce passage, debout à la fenêtre, contemplant le monde extérieur, attendant l'arrivée d'un ami qui n'arrivera pas, revient comme une hantise plusieurs fois au cours du roman, à la manière d'un refrain.

La fenêtre est non seulement ce qui permet l'observation de l'extérieur mais représente le passage par lequel les menaces de l'extérieur peuvent arriver. Dans ces extraits où la narratrice se tient debout devant la fenêtre elle hésite au seuil entre l'intérieur et l'extérieur, en songeant « à la joie imminente de revoir [s]on copain, une joie que [s] on père ne semblait pas admettre » $(50)$. Le père, conscient du fait que la fenêtre relie l'extérieur et l'intérieur dans les deux sens, veut fermer cette fenêtre car il sait que, si les éléments extérieurs peuvent pénétrer dans l'intimité de la maison (l'air, l'ami que la narratrice attend), la fenêtre peut aussi laisser échapper des choses de l'intérieur vers l'extérieur, notamment sa fille tant aimée, dont «l'être entier » est «penché » vers l'extérieur (88).

La fenêtre, en plus d'être l'espace où intérieur et extérieur se confondent, s'apparente également aux peintures du père, et par extension, à la création artistique. Au moment où $\mathrm{A}$. vient chercher la narratrice à la maison du père, la narratrice dit que la fenêtre, «par sa fraîcheur temporaire mais récurrente confondra le dehors et le dedans, le clair et l'obscur », cependant, « [1]a lumière change si vite que, en un instant, la fenêtre ne paraîtra plus la même, et qu'il sera impossible de la peindre » (125-26). Puisque la fenêtre est également un tunnel, une aire de jeu, elle s'accompagne d'une remise en question permanente qui se traduit par des fluctuations perpétuelles dans la luminosité. Ceci rend la représentation artistique quasiment impossible, selon la narratrice, qui voit la peinture comme une tentative de fixer l'état de la fenêtre dans un moment précis et défini dans le temps. 
Cependant, au lieu d'être des œuvres d'art qui fixent les sujets dans un état particulier, les sujets des tableaux du père ne sont pas fixes et résistent à toute catégorisation, comme le jeu de lumière décrit dans les dernières lignes du roman. Les sujets des tableaux du père « sont difficiles à saisir, les lignes tordues, ambiguës, l'homme et l'animal souvent superposés ou confondus » (23). Flous, entre l'humain et l'animal, ces sujets rappellent le père et la fille qui sont les descendants d'un poisson. Le père, dans ses tableaux, ne fixe pas ses sujets dans un rôle ou dans un état fixe, sans se soucier des catégories conventionnelles comme l'humain et l'animal.

La singularité des tableaux du père s'illustre par le choix des tableaux à apparaître au vernissage auquel la narratrice assiste au début du roman. Les experts chargés de faire le tri dans les tableaux du peintre disparu ne s'intéressent qu'aux œuvres où la distinction entre l'animal et l'humain est claire et les sujets relativement faciles à saisir. Dans la maison abandonnée, la narratrice trouve un tableau laissé par les experts. Le sujet de ce tableau a «le cou [...] exagérément gonflé », comme s'il «est en train d'avaler quelque chose de gros » (99). La narratrice comprend que les experts y ont certainement vu un «détail extravagant» qui pourrait décevoir ou même repousser le public potentiel.

$\mathrm{Au}$ cours du roman, un système d'équivalences s'établit entre le père et l'artiste d'une part et la fille et l'œuvre produite d'autre part. Les tableaux deviennent ce tunnel, cette aire de jeu où père et fille, création et œuvre, se confondent. C'est l'atmosphère des tableaux du peintre présentés au vernissage qui sert de catalyse, pour la narratrice, à la mémoire de la vie passée puisqu'elle reconnaît « tout de suite [...] l'atmosphère de cet insolite après-midi où [...] l'œuvre s'était confondue pour de bon avec le créateur » (34). L'après-midi dont elle se souvient est celui du moment où elle se fait avaler par son père et fait un saut dans le temps et dans l'espace pour arriver dans l'espace-temps de A. Comme la fenêtre et le ventre qui se confondent dans le récit de la narratrice, le créateur et l'œuvre font à la fois allusion aux tableaux et à l'artiste et à la fille en tant que la création du père.

L'image du peintre qui se confond avec son œuvre indique un lien dans le texte avec le taoïsme. Puisque la narratrice «supposai[t] que toutes les réalités [du corps du père] [...] étaient représentées dans sa peinture de façon concentrée », elle imagine son père « reculant vers le fond du salon, sa figure se confondant lentement avec celle du tableau » $(50,93)$. Le père représente son corps dans les tableaux de manière si efficace qu'il devient impossible de distinguer le corps 
de l'œuvre artistique, le corps du corpus. Dans sa discussion du rôle du taoïsme dans la nouvelle «Comment Wang-Fô fut sauvé » de Marguerite Yourcenar ${ }^{3}$, Tibor Polgár note que «chez les peintres taoïstes, tout le processus de travail découle d'une observation détaillée, presque d'une unification harmonieuse avec l'objet » (81). Quand elle revient à la maison du père, la narratrice ne retrouve pas de trace de son père, détail qui semble troubler l'inspecteur également. Dans cette confusion ultime entre l'artiste et son œuvre, le corps du père s'unifie avec l'objet, comme chez les peintres taoïstes, même s'il ne s'agit pas précisément d'une unification harmonieuse.

Le lien avec le taoïsme permet également d'identifier la pertinence de l'image du tunnel au rapport entre le créateur et l'œuvre. À la différence de l'appareil digestif ou de la fenêtre, il n’y a pas d'espace où le tunnel se situe. Polgár signale un chapitre du Tao Te King qui partage de fortes ressemblances avec le tunnel de la création décrit par Ying Chen : «Sans sortir de ma maison, je connais l'univers; sans regarder par la fenêtre, je découvre les voies du ciel » (Livre II 47). La description de la «pièce grise » de l'écrivain du tunnel fait écho à ces vers. Dans le cas du créateur qui se confond avec son œuvre, le tunnel est le processus de création par la remise en question des frontières qui aboutit à une confusion parfaite entre créateur et œuvre.

L'univers du Mangeur est un univers où chaque frontière, chaque catégorie est systématiquement remise en question. Ces frontières s'effritent dans les tunnels de l'espacetemps de A., le ventre de ce dernier, ses tableaux, et la fenêtre. La frontière entre le passé et le présent est tellement faible que la narratrice passe entre les deux sans s'en rendre compte tandis que le tunnel de l'appareil digestif permet de repenser la relation entre le mangeur et la proie comme un processus de collaboration aboutissant à un changement d'état et non pas comme un acte sauvage d'anthropophagie. Ce changement d'état s'accompagne de l'image de la fenêtre, qui relie non seulement l'extérieur et l'intérieur, mais se trouve être un lieu privilégié où tous les tunnels se juxtaposent. Cette remise en question persistante des frontières, comprise dans le contexte du jeu défini par Hans et Küchler et qui prend la forme du tunnel chez Ying Chen, est une quête de sens et un processus de création qui est, par définition, sans fin, continuellement « à suivre $»^{4}$. La fin idéale est celle qui n'est possible que dans la fiction, celle d'une confusion entre créateur et œuvre, détruisant la frontière ultime opposant l'artiste et ses créations. 
Voix plurielles 10.2 (2013)

\section{Ouvrages cités}

Chen, Ying. Espèces. Montréal : Boréal, 2010.

---. Immobile. Montréal : Boréal, 1998.

---. La mémoire de l'eau. Montréal : Leméac, 1992.

---. La rive est loin. Montréal : Boréal, 2013.

---. Le champ dans la mer. Montréal : Boréal, 2002.

---. Le mangeur. Paris : Seuil, 2005.

---. «Le tunnel ». Alibi 2 : Dialogues littéraires franco-chinois. Ed. Annie B. Curien. Paris :

Maison des sciences de l'homme, 2005. 118-20.

---. Les lettres chinoises. Montréal : Leméac, 1993.

---. L'ingratitude. Montréal : Leméac, 1995.

---. Quatre mille marches : un rêve chinois. Paris : Seuil, 2004.

---. Querelle d'un squelette avec son double. Montréal : Boréal, 2003.

---. Un enfant à ma porte. Montréal : Boréal, 2008.

---. « Rencontre littéraire avec Ying Chen ». Congrès 2013 de la Fédération canadienne des sciences humaines. U de Victoria. (2 juin 2013).

Hans, James S. The Play of the World. Amherst : U of Massachusetts P, 1981.

Hearn, Lafcadio. A Japanese Miscellany. Boston : Little, Brown, 1901.

Henriot, Jacques. Sous couleur de jouer. Paris : Corti, 1989.

Inaga, Shigemi. «The Painter Who Disappeared in the Novel : Images of an Oriental Artist in European Literature ». Text and Visuality : Word and Image Interactions 3. Ed. Martin Heusser. Atlanta : Rodopi, 1999. 117-27.

Küchler, Tilman. Postmodern Gaming : Heidegger, Duchamp, Derrida. New York : Lang, 1994.

Parker, Gabrielle. «À mi-chemin entre deux mondes : parcours féminins chez Ying Chen ». RELIEF-Revue électronique de littérature française 5.2 (2011) : 75-87. <http://www.revue-relief.org/index.php/relief/article/view/690/757>.

Polgár, Tibor. «Une vision du monde entre l'Occident et le tao dans la nouvelle 'Comment Wang-Fô fut sauvé' de Marguerite Yourcenar ». Tableaux Changeants. Dir. Kata Gyuris. Budapest: Collegium Eötvös Jósef, 2013. 77-88. 
Silvester, Rosalind. «Ying Chen and the 'Non-Lieu' ». The Modern Language Review 106.2

(2011) : 407-22.

« Tao Te King ». Tao Te King, livre de la voie et de la vertu. Tr. Julien Stanislas. N.p., n.d. http://taoteking.free.fr/index.html (11 Nov. 2013).

Yourcenar, Marguerite. Nouvelles orientales. Paris : Gallimard, 1981.

NOTES

1 L'existence de ce petit texte souvent absent des discussions sur Ying Chen contenant une métaphore puissante pour la création chez cette dernière m'a été signalée dans l'article «À mi-chemin entre deux mondes... » de Gabrielle Parker.

2 Le mot «manger » en français porte une forte connotation sexuelle et, ce, depuis le dix-neuvième siècle. Il existe entre le père et la fille dans Le mangeur une certaine tension sexuelle qui pourrait faire l'objet d'une étude plus approfondie. Notre étude se concentre sur l'identité de la narratrice définie à travers le saut, le passage entre deux espaces-temps. Dans le contexte de cette étude, donc, nous examinerons le rôle de l'estomac comme lieu symbolique de transformation et non pas de consommation sexuelle.

3 Cette nouvelle figure également un artiste qui se confond avec son œuvre. Au lieu de prendre sa source dans une légende chinoise, cependant, il s'agirait d'une adaptation d'un conte japonais traduit en anglais par l'écrivain irlandais Lafcadio Hearn sous le titre «The Story of Kwashin Koji » (Shigemi 117).

4 Lors de la « Rencontre littéraire avec Ying Chen », l'écrivain présente La rive est loin (2013) comme la fin de la série d'épisodes partageant tous la même narratrice. Il sera donc nécessaire d'étudier place de la figure du tunnel dans ce dernier roman par rapport à l'idée de la fin, qui n'est pas présente dans les romans précédents. 